\title{
Initiation gait variability is higher in the morning in elderly inpatients
}

\author{
Yosuke IshII, PT, $\mathrm{PhD}^{1,2}$, Yuichiro KAI, $\mathrm{MD}^{3}$, Takashi MoRITA, $\mathrm{PT}^{1}$, \\ Hisayoshi AIKAWA, $\mathrm{PT}^{1}$ and Ryoichi NAKAMURA, $\mathrm{PT}^{1}$ \\ ${ }^{1)}$ Department of Rehabilitation, Medical Technology, Shimura Hospital \\ ${ }^{2}$ Health Sciences Major, Graduate School of Biomedical \& Health Sciences, Hiroshima University \\ ${ }^{3)}$ Department of Surgery, Shimura Hospital
}

\begin{abstract}
Objective: Although elderly inpatients are known to experience decreased physical activity in the morning, falls occur frequently during this time. Gait variability is an evaluation of gait instability and a risk factor for falls. Gait initiation requires complex processes, and it is important to evaluate gait variability not only during steady-state gait but also during gait initiation. However, the effect of the diurnal pattern on variability in gait characteristics is still unknown. The aim of this study was to investigate the effect of the diurnal pattern on initiation and steady-state gait variability in elderly inpatients. Method: Thirty-seven elderly inpatients ( 28 women; mean age, $79.7 \pm 9.5$ years) who could walk without support were sampled in this study. The quantitative measure of gait variability was evaluated using the coefficient of variation (CV) based on four consecutive stride durations determined using triaxial accelerometers. Gait characteristics were evaluated during initiation and steady-state gait and defined as initiation CV and steady-state CV, respectively. This measurement was performed at two time points, morning and daytime. Results: There was no significant difference between initiation and steady-state gait characteristics in the daytime condition. However, in the morning condition, the initiation CV was higher than the steady-state CV. Furthermore, the initiation $\mathrm{CV}$ was higher in the morning than during daytime $(\mathrm{p}<0.01)$. Conclusion: Our study revealed that the variability of initiation gait is higher in the morning. It may be important to assess the risk of falls, including initiation gait, in the morning.
\end{abstract}

Key words: Elderly inpatient, gait variability, initiation gait, diurnal pattern

(Phys Ther Res 23: 160-165, 2020)

\begin{abstract}
A ging causes poor balance secondary to neuromuscular changes $^{1,2)}$. Falls often occur in elderly people with poor balance, and they are a risk factor for bone fractures ${ }^{3,4)}$. Falls can lead to severe disability in elderly inpatients, resulting in delayed treatment and longer hospital stays ${ }^{5-7)}$. Thus, prevention of falls is important to promote the smooth treatment of elderly inpatients.

Although physical activity is known to be reduced in the morning, the incidence of falls during this time is higher than that during daytime ${ }^{8,9)}$. This suggests that specific

Received: December 13, 2019

Accepted: April 27, 2020

Advance Publication by J-STAGE: July 22, 2020

Correspondence to: Yosuke Ishii, Shimura Hospital, 3-13 Funairicho, Naka-Ku, Hiroshima 730-0841, Japan

\# e-mail: s.h.iypt@gmail.com

doi: 10.1298/ptr.E10019
\end{abstract}

changes associated with falls may occur during the morning. Several studies have reported poor postural control in the morning compared with that during the daytime ${ }^{10,11)}$. However, they measured static postural control and not dynamic control. Falls often occur during unstable walking. Although the evaluation of dynamic postural control during gait is important for the prevention of falls, the effect of the diurnal pattern on dynamic postural control during gait is not well understood.

Gait unintentionally fluctuates with each stride, and gait variability is known to reflect poor balance during ambulation $^{12)}$. Many studies have reported that high gait variability is associated with fall incidence in elderly individuals ${ }^{12,13)}$. Moreover, Mbourou et al. investigated the gait characteristic of a faller and non-faller and reported that gait variability of the initial strides was higher in fallers when compared with that in non-fallers ${ }^{14)}$. Their results suggest 
Table 1. Demographic data of participants.

\begin{tabular}{lcccc}
\hline & Inpatient & Orthopedic & Stroke & $\mathrm{p}$ \\
\hline Participants & 37 & 26 & 11 & \\
Gender (M:F) & $9: 28$ & $2: 24$ & $7: 4$ & \\
Age (years) & $79.7 \pm 9.5$ & $81.5 \pm 7.7$ & $76.1 \pm 11.1$ & 0.108 \\
Height $(\mathrm{cm})$ & $149.8 \pm 10.5$ & $147.9 \pm 10.0$ & $154.4 \pm 9.9$ & 0.087 \\
Weight $(\mathrm{kg})$ & $52.1 \pm 10.2$ & $49.9 \pm 10.0$ & $56.9 \pm 7.4$ & 0.051 \\
BMI (kg/m²) & $23.0 \pm 3.0$ & $22.5 \pm 3.5$ & $23.7 \pm 1.3$ & 0.299 \\
Post-injury (day) & $69.2 \pm 36.8$ & $58.7 \pm 34.3$ & $94.2 \pm 27.6$ & 0.005 \\
\hline
\end{tabular}

BMI, Body mass index. Values represent mean \pm standard deviation. The p-value relates to comparisons between the orthopedic and stroke subgroups.

that the initiation gait requires complex processes and may reveal important information associated with the risk of falls. However, some previous studies that analyzed gait variability only examined the steady-state gait condition and excluded the first few strides ${ }^{15,16}$. Although it is essential to investigate both steady-state gait and initiation gait for an understanding of gait variability, the effect of the diurnal pattern on the variability in each gait process is still unknown.

The aim of this study was to investigate the diurnal variation in initiation and steady-state gait variability in elderly inpatients. This study will be useful in preventing falls in elderly inpatients. We hypothesized that elderly inpatients have a high variability of initiation gait in the morning relative to that during daytime.

\section{Material and Methods}

\section{Participants}

Thirty-seven elderly inpatients who could walk were sampled in this study (inpatient group: mean age, $79.7 \pm$ 9.5 years). The inpatient group included orthopedic and stroke patients (orthopedic: $70 \%$ ) and were subdivided into orthopaedic and stroke subgroups. The orthopedic subgroup included trauma fractures (hip $42 \%$, spine $38 \%$, pelvis $4 \%$, and lower leg $8 \%$ ) according to fall events, and knee osteoarthritis (8\%), and almost all patients had undergone surgical treatment $(85 \%)$. On the other hand, the stroke subgroup included infarction (38\%) and hemorrhage $(72 \%)$ and had moderate paralysis of the upper and lower limbs (Brunnstrom stage, upper: $4.5 \pm 1.2$; lower: $4.7 \pm$ $0.9)$. Demographic data are shown in Table 1. Patients were excluded if they had diseases that could directly affect balance, such as chronic head injuries, low blood pressure, vestibular conditions, diabetic peripheral sensory disorder, severe dementia, or Parkinson's disease/parkinsonism.

Each participant provided informed consent prior to participating in this study. This study was approved by the ethics committees of Shimura Hospital (approval number: 22) according to the Helsinki Declaration.

\section{Functional physical assessment}

All patients were evaluated using the functional independence measure (FIM). The FIM is scored based on activity limitations and performance observations. A high score reflects independence in daily life.

Balance ability was determined using the timed up and go test (TUG). This test evaluates balance during motions, including sit-to-stand, walking $3 \mathrm{~m}$, and turning around. Participants were asked to perform a series of actions as quickly as possible and informed that the increased time to perform the task was undesirable. This functional physical data was evaluated at once during the daytime.

\section{Assessment of gait variability}

An inertial motion sensor, including triaxial accelerometers and gyrosensor (WAA-010, ATR-Promotions, Japan), was placed on the lower back to be close to the body's center of mass according to the lumbar spinal process at the level of third based on a previous study ${ }^{32}$ and attached using a belt. The data were collected during a 10-m walk at a $100-\mathrm{Hz}$ sampling rate. To reflect the natural walking, the patients were asked to walk with a comfortable speed once and the first step was not instructed on which leg when they started walking. All data were analyzed using MATLAB software (MATLAB 2015a, MathWorks, Japan). The raw acceleration data were processed using a low-pass filter with a cut-off frequency of $10 \mathrm{~Hz}$. The time for one stride was determined based on the timing of the heel strike, which produced peak acceleration in the anteroposterior direction ${ }^{17)}$. The quantitative value of gait variability was defined as the coefficient of variation (CV) based on four consecutive stride durations determined using triaxial accelerometers and was calculated using the formula (standard deviation [SD] of stride time/average stride time) $\times 100$. The $\mathrm{CV}$ was evaluated for initiation and steady-state gait, referred to as initiation $\mathrm{CV}$ and steady-state $\mathrm{CV}$, respectively. As the representative value for statistical analyses, in the initiation $\mathrm{CV}$, the leg with the first stride, and in the steady-state $\mathrm{CV}$, one leg with a higher CV in both legs was adopted. The starting point of the strides for initiation and steady state were determined as the first and middle 
Table 2. Functional physical assessment in each subgroup

\begin{tabular}{lcccc}
\hline & Inpatient & Orthopedic & Stroke & $\mathrm{p}$ \\
\hline Gait speed (m/s) & $0.7 \pm 0.2$ & $0.8 \pm 0.2$ & $0.7 \pm 0.3$ & 0.225 \\
TUG (sec) & $17.8 \pm 11.0$ & $15.1 \pm 7.1$ & $24.1 \pm 14.7$ & 0.019 \\
FIM (score) & $111.1 \pm 12.4$ & $112.2 \pm 11.3$ & $108.4 \pm 14.0$ & 0.403
\end{tabular}

TUG, timed up and go test; FIM, functional independence measure. Values represent mean \pm standard deviation. The p-value relates to comparisons between the orthopedic and stroke subgroups.

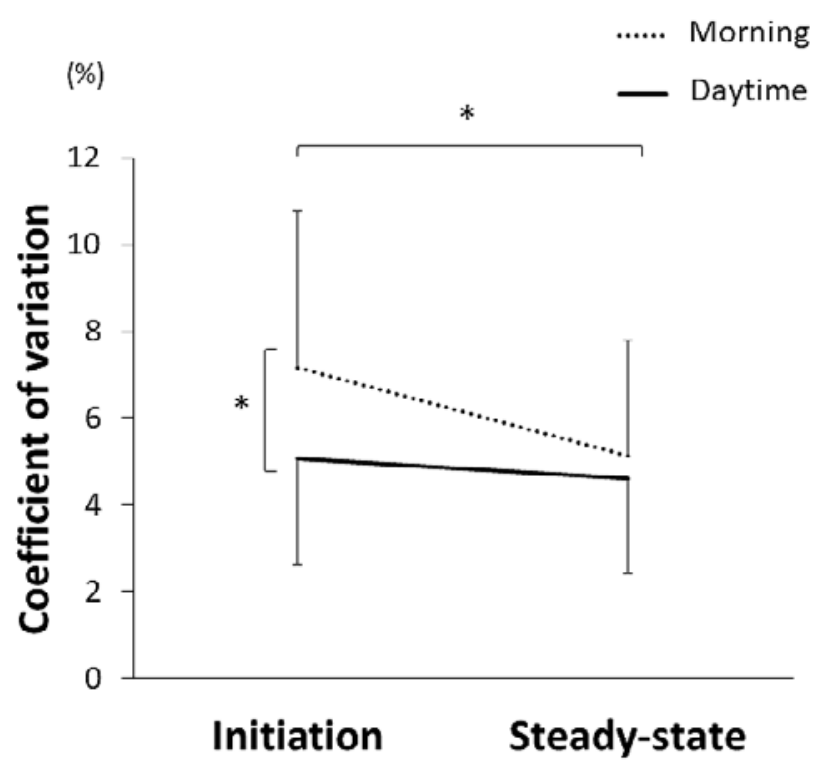

Figure 1. The characteristic gait variability between-condition.

Values represent means \pm standard deviation. *significant difference between gait characteristics and conditions, $\mathrm{p}<0.01$.

points during walking. The evaluation of gait variability was randomly performed in two time conditions: morning (between 7:00 and 8:00 a.m.) and daytime (between 1:00 and 2:00 p.m.).

\section{Statistical analyses}

The functional physical assessments and demographic data were compared in each subgroup using the Student's ttest. The Shapiro-Wilk test demonstrated heterogeneity in age, height, FIM, and TUG, and these were analyzed using the Mann-Whitney $U$ test. To analyze the difference in gait variability between time of day and gait process in elderly patients, a two-way factorial analysis of variance with replication was performed. Additionally, to analyze the gait variability in each subgroup, a three-way factorial analysis of variance with replication was conducted. These analyses were followed by Bonferroni correction as post hoc testing. All statistical analyses were performed using R, i386, version 3.4.1. The significance level was set at $5 \%$.

\section{Results}

\section{Participants' demographic and functional physical as-} sessment data

The demographic data of the participants are shown in Table 1 . The post-injury day was significantly longer in stroke patients, but the other data showed no significant differences between subgroups (Table 1).

Moreover, the FIM score and gait speed were not different between subgroups (Table 2). However, the time taken for TUG in stroke patients was significantly greater than that in orthopedic patients. (Table 2).

\section{Comparison of characteristic gait variability between conditions in the inpatient group}

There was an interaction between the gait process and condition $(F(1,36)=7.59, \mathrm{p}<0.01)$. On the main effects, the $\mathrm{CV}$ in the morning was significantly higher than that during the daytime (condition: $F(1,36)=14.36, \mathrm{p}<0.01$ ). Moreover, the value of the initiation $\mathrm{CV}$ in the morning was significantly higher than that during the daytime (gait process: $F(1,36)=14.46, \mathrm{p}<0.01)$.

With regard to gait process, in the morning condition, the initiation $\mathrm{CV}$ was significantly higher than the steadystate CV $(\mathrm{p}<0.01)$. On the other hand, there was no significant difference in the $\mathrm{CV}$ value for gait process in the daytime condition (Figure 1).

3. Comparison of characteristic gait variability between conditions in each subgroup

There was interaction among the gait process, condition, and group $(F(1,35)=6.0, \mathrm{p}<0.05)$. The main effects for gait process, condition and group were found by repeated-measures analysis (gait process: $F(1,35)=17.91$, p $<0.01$; condition: $F(1,35)=24.9, \mathrm{p}<0.01)$; group : $F$ $(1,35)=5.3, \mathrm{p}<0.05)$.

In the morning condition, the initiation $\mathrm{CV}$ in stroke inpatients was significantly higher than that in orthopedic patients $(\mathrm{p}<0.01)$. However, the steady-state $\mathrm{CV}$ in the morning condition, and initiation and steady-state in daytime were not different between subgroups.

With regard to gait process and conditions, in both groups, the initiation $\mathrm{CV}$ was higher than steady-state $\mathrm{CV}$ in the morning but not during daytime (orthopedic: p < 

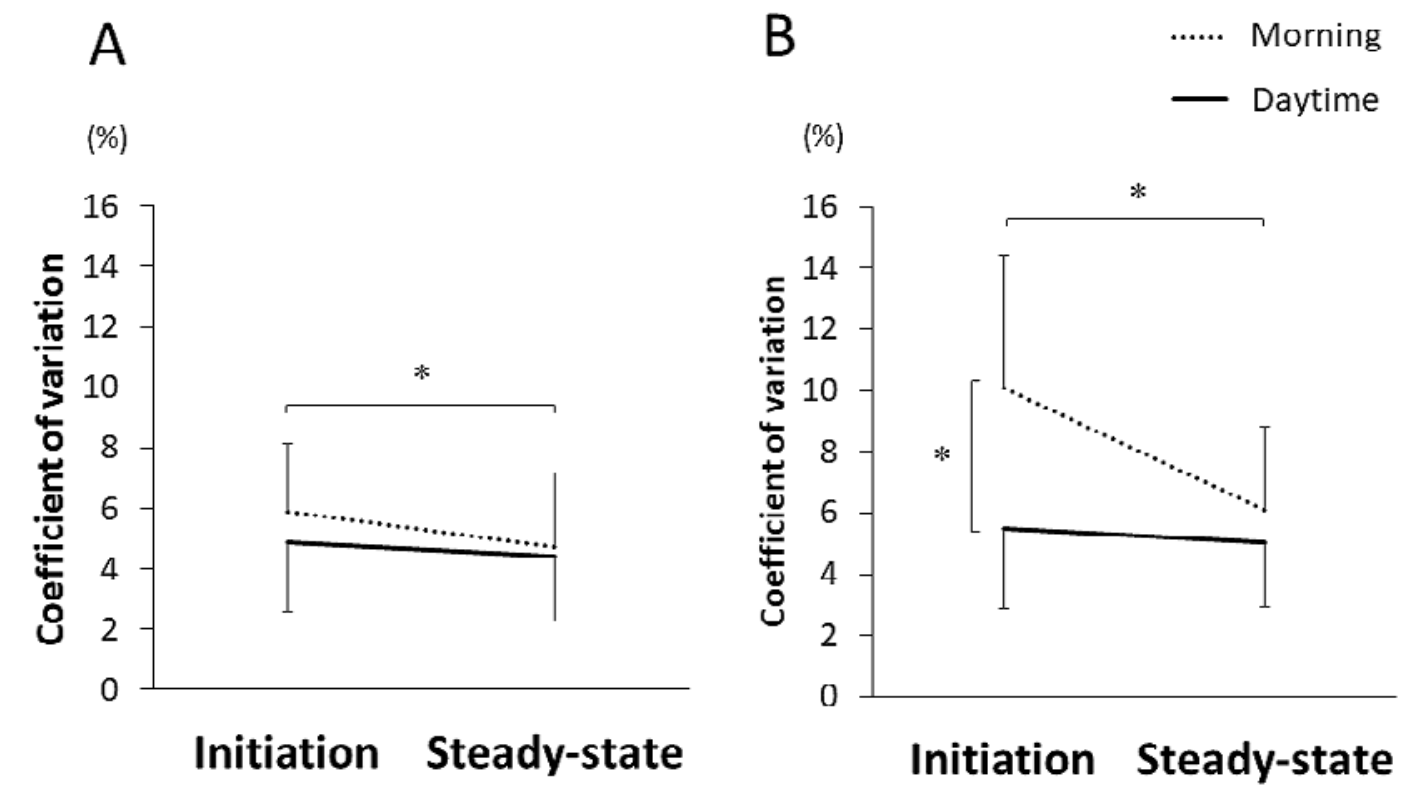

Figure 2. The characteristic gait variability between-condition in each subgroup.

Orthopedic (A), stroke patients (B). Values represent means \pm standard deviation. *significant difference between gait characteristics and conditions, $\mathrm{p}<0.05$.

0.05, stroke: $\mathrm{p}<0.01$ ) (Figure 2A, B). Moreover, in stroke inpatients, the initiation $\mathrm{CV}$ in the morning condition was significantly higher than that in the daytime condition $(\mathrm{p}<$ 0.01) (Figure 2B).

\section{Discussion}

Our study found that initiation gait had greater variability than steady-state gait, and this phenomenon was particularly apparent in the morning, especially in elderly inpatients with stroke. These findings show that elderly inpatients have a higher risk of falling during gait initiation in the morning than during daytime.

Hausdorff et al. investigated the differences in gait variability of the stride time among young, non-faller elderly, and faller elderly in the community-dwelling subjects $^{33)}$. Their results showed a higher CV in fallers (young: $1.8 \%$, non-faller elderly: $2.3 \%$, faller elderly: $4.2 \%$ ), suggesting that the obtained higher $\mathrm{CV}$ was reflected as a characteristic of the elderly faller. In the present study, the obtained CV was $5.4 \%$, which is similar to the fallen elderly, and might indicate that our participants also have the characteristics of fallers based on a previous study.

In elderly inpatients, we confirmed that the initiation $\mathrm{CV}$ was higher in the morning condition, although steadystate CV was not significantly different between the two conditions. Additionally, the initiation CV was higher than steady-state CV only in the morning condition. These results support our hypothesis and might indicate that elderly inpatients have an unstable gait pattern at the initiation of walking in the morning. Several studies have reported that high CV during gait is associated with poor postural con- trol $^{18,19)}$. Moreover, many studies of diurnal patterns have shown that postural control ability fluctuates with time and declines in the morning ${ }^{20-22)}$. However, another study evaluated postural control and reported no difference in the ability in the morning and daytime ${ }^{23,24)}$. Therefore, these previous studies indicate that the effect of time alone on postural control may not be clearly present. However, gait variability increases when performing dual tasks compared to a single task, indicating that the required high level of postural control increases gait variability ${ }^{25,26}$. Regarding gait process, a difference in postural control between the initial gait and steady gait has been reported; initial gait requires greater integration and coordination of multiple sensory modalities such as visual, vestibular, and somatosensory senses ${ }^{27)}$. These previous studies show that initial gait is more affected by the diurnal pattern because of the required higher ability, which might explain why the variation in initial gait was higher in the morning.

Additionally, the phenomenon of higher initiation gait variability in the morning was clearer in stroke patients than in orthopedic patients. The initial gait variability in the morning may be related to patient characteristics. Many studies have reported higher gait variability in stroke patients $^{28,29)}$. Callisaya et al. investigated the association between sensorimotor ability and high gait variability and reported the correlation with poor body sway, reaction time, and proprioception function ${ }^{18)}$. These previous studies indicate that gait variability, including irregular foot placement, may cause a lack of postural control due to sensorimotor disorders. In the present study, we used TUG, which is associated with sensorimotor abilities ${ }^{30)}$, and observed that stroke patients had a longer TUG time than orthopedic pa- 
tients. Additionally, a previous study reported that postural control due to sensorimotor ability was poor in the morning when compared with that in the daytime because of the worse symptoms $^{31)}$. In this study, stroke patients with moderate paralysis might also have worsened symptoms such as spasms and sensorimotor in the morning. These results and previous studies support the validity of our findings that gait variability in the morning is a characteristic of patients with sensorimotor disorders.

We evaluated gait stability using the variability of the gait cycle and showed that the value of variability gait is higher during the few initial strides in the morning. Falls are believed to be caused by an irregular motion that differs from a person's intention in a dynamic condition. A previous study reported that elderly individuals with a history of falls exhibited higher gait variability ${ }^{14}$. Moreover, another study revealed that high gait variability was associated with the incidence of falls in elderly inpatients ${ }^{26)}$. These studies indicate that higher gait variability is a risk factor for falls, and its management is important for preventing falls. In the present study, we revealed that initiation gait influenced gait variability in the morning. Therefore, evaluation of gait variability, including gait initiation, should be better able to identify the risk of falls in inpatients and help prevent falls in the morning.

There were several limitations to this study. First, our sample included inpatients with a variety of characteristics, making it difficult to analyze patient characteristics and obtain detailed information such as the nature of falls. For example, some patients had a history of falls; therefore, fall risk might have been affected by psychological factors in these patients. Second, this study did not acquire sleep quality data. A previous study reported an association between sleep quality and gait variability and suggested that low quality sleep affects gait performance ${ }^{34)}$. Third, patients took different medications and were treated using different rehabilitation approaches; these factors can influence gait performance. Future studies need to acquire data regarding sleep quality in participants with matched pathological states.

\section{Conclusion}

Our study revealed that initiation gait influenced gait variability in the morning, especially in elderly inpatients with stroke. In evaluations aiming to prevent falls, it may be particularly important to assess the initiation gait in the morning.

\section{Conflict of Interest: None.}

Funding sources: This research did not receive any specific grant from funding agencies in the public, commercial, or not-for-profit sectors.
Acknowledge: The authors thank Hiroshi Kurumadani, Graduate School of Biomedical \& Health Sciences, Hiroshima University, for providing support for measurement.

\section{References}

1) Wingert JR, Welder C, et al.: Age-Related Hip Proprioception Declines: Effects on Postural Sway and Dynamic Balance. Arch Phys Med Rehabil. 2014; 95: 253-261.

2) Osoba MY, Rao AK, et al:: Balance and gait in the elderly: A contemporary review. Laryngoscope Investig Otolaryngol. 2019; 4: 143-153.

3) Dargent-Molina P and Bréart G: Epidemiology of falls and fallrelated injuries in the aged. Rev Epidemiol Sante Publique. 1995; 43: 72-83.

4) Baldursdottir B, Petersen H, et al: : Sensory impairments and wrist fractures: A case-control study. J Rehabil Med. 2018; 50: 209-215.

5) Hars M, Audet M-C, et al.: Functional Performances on Admission Predict In-Hospital Falls, Injurious Falls, and Fractures in Older Patients: A Prospective Study. J Bone Miner Res Off J Am Soc Bone Miner Res. 2018; 33: 852-859.

6) Gacto-Sánchez M, Medina-Mirapeix F, et al.: Changes in disability levels among older adults experiencing adverse events in postacute rehabilitation care: a prospective observational study. Medicine (Baltimore). 2015; 94: e570.

7) Hill A-M, Jacques A, et al.: In-Hospital Sequelae of Injurious Falls in 24 Medical/Surgical Units in Four Hospitals in the United States. Jt Comm J Qual Patient Saf. 2019; 45: 91-97.

8) Lehtola S, Koistinen P, et al.: Falls and injurious falls late in home-dwelling life. Arch Gerontol Geriatr. 2006; 42: 217-224.

9) Cantwell K, Burgess S, et al.: Temporal trends in falls cases seen by EMS in Melbourne: The effect of residence on time of day and day of week patterns. Injury. 2016; 47: 266-271.

10) Sato N, Yamamoto R, et al.: Diurnal Variation in the Static Balance of Hospitalized Patients. Rigakuryoho Kagaku. 2016; 31 : 451-454.

11) Bougard C and Davenne D: Morning/Evening differences in somatosensory inputs for postural control. BioMed Res Int. 2014; 2014: 287436.

12) Hausdorff JM, Rios DA, et al.: Gait variability and fall risk in community-living older adults: a 1-year prospective study. Arch Phys Med Rehabil. 2001; 82: 1050-1056.

13) Hausdorff JM: Gait dynamics, fractals and falls: Finding meaning in the stride-to-stride fluctuations of human walking. Hum Mov Sci. 2007; 26: 555-589.

14) Mbourou GA, Lajoie Y, et al.: Step length variability at gait initiation in elderly fallers and non-fallers, and young adults. Gerontology. 2003; 49: 21-26.

15) Lindemann U, Najafi B, et al.: Distance to achieve steady state walking speed in frail elderly persons. Gait Posture. 2008; 27 : 91-96.

16) Najafi B, Miller D, et al.: Does footwear type impact the number of steps required to reach gait steady state?: an innovative look at the impact of foot orthoses on gait initiation. Gait Posture. 
2010; 32: 29-33.

17) Zijlstra W and Hof A: Assessment of spatio-temporal gait parameters from trunk accelerations during human walking. Gait Posture. 2003; 18: 1-10.

18) Callisaya ML, Blizzard L, et al.: Sensorimotor factors affecting gait variability in older people--a population-based study. J Gerontol A Biol Sci Med Sci. 2010; 65: 386-392.

19) Jayakody O, Breslin M, et al.: Medical, Sensorimotor and Cognitive Factors Associated With Gait Variability: A Longitudinal Population-Based Study. Front Aging Neurosci. 2018; 10: 419.

20) Forsman $P$ and Hæggström E: Circadian amplitude and homeostatic buildup rate in postural control. Gait Posture. 2013; 38: 192-197.

21) Sørensen RR, Jørgensen MG, et al.: Impaired postural balance in the morning in patients with knee osteoarthritis. Gait Posture. 2014; 39: 1040-1044.

22) Matsuura A, Tomimura K, et al.: Time-of-Day Effects on Dynamic Postural Control. Rigakuryoho Kagaku. 2019; 34: 79-82.

23) Jorgensen MG, Rathleff MS, et al.: Time-of-day influences postural balance in older adults. Gait Posture. 2012; 35: 653-657.

24) Gribble PA, Tucker WS, et al.: Time-of-day influences on static and dynamic postural control. J Athl Train. 2007; 42: 35-41.

25) Hamacher D, Hamacher D, et al.: Effect of dual tasks on gait variability in walking to auditory cues in older and young individuals. Exp Brain Res. 2016; 234: 3555-3563.

26) Kressig RW, Herrmann FR, et al.: Gait variability while dual- tasking: fall predictor in older inpatients? Aging Clin Exp Res. 2008; 20: 123-130.

27) Yiou E, Caderby T, et al.: Balance control during gait initiation: State-of-the-art and research perspectives. World J Orthop. 2017; 8: 815-828.

28) Goto S, Yasuyama I, et al.: Assessment of Gait Stability in Stroke Patients. Rigakuryoho Kagaku. 1997; 12: 57-61.

29) Reissman ME and Dhaher YY: A functional tracking task to assess frontal plane motor control in post stroke gait. J Biomech. 2015; 48: 1782-1788.

30) Kwan MM-S, Lin S-I, et al.: Sensorimotor function, balance abilities and pain influence Timed Up and Go performance in older community-living people. Aging Clin Exp Res. 2011; 23: 196-201.

31) Zhang Z, Lion A, et al.: Diurnal variation on balance control in patients with symptomatic knee osteoarthritis. Arch Gerontol Geriatr. 2015; 61: 109-116.

32) Auvinet B, Touzard G, et al.: Reference data for normal subjects obtained with an accelerometric device. Gait Posture. 2002; 16: 124-134.

33) Hausdorff JM, Edelberg HK, et al:: Increased gait unsteadiness in community-dwelling elderly fallers. Arch Phys Med Rehabil. 1997; 78: 278-283.

34) Agmon M, Shochat T, et al.: Sleep quality is associated with walking under dual-task, but not single-task performance. Gait Posture. 2016; 49: 127-131. 